\title{
Impact of improved neonatal care on the profile of retinopathy of prematurity in rural neonatal centers in India over a 4-year period
}

This article was published in the following Dove Press journal:

Eye and Brain

20 May 2016

Number of times this article has been viewed

\author{
Anand Vinekar' \\ Chaitra Jayadev' \\ Siddesh Kumar ${ }^{2}$ \\ Shwetha Mangalesh ${ }^{1,3}$ \\ Mangat Ram Dogra ${ }^{4}$ \\ Noel J Bauer ${ }^{5}$ \\ Bhujang Shetty ${ }^{6}$
}

'Department of Pediatric Retina, Narayana Nethralaya Eye Hospital, Bangalore, ${ }^{2}$ Raichur Institute of Medical Sciences, Raichur, Karnataka, India; ${ }^{3}$ Department of Ophthalmology, Duke University, Durham, NC, USA; ${ }^{4}$ Advanced Eye Center, Postgraduate Institute of Medical Education and Research, Chandigarh, India; ${ }^{5}$ Department of Ophthalmology, Maastricht University of Health Sciences, Maastricht, the Netherlands; ${ }^{6}$ Department of Ophthalmology, Narayana Nethralaya Postgraduate Institute of Ophthalmology, Bangalore, India
Correspondence: Anand Vinekar Department of Pediatric Retina, Narayana Nethralaya Eye Hospital, I2 I/C, Chord Road, Ist 'R' Block, Rajajinagar, Bangalore 560 0I 0, Karnataka, India Fax +9l 8023377329

Email anandvinekar@yahoo.com
Purpose: To report the reduction in the incidence and severity of retinopathy of prematurity (ROP) in rural India over a 4-year period following the introduction of improved neonatal care practices.

Methods: The Karnataka Internet Diagnosis of Retinopathy of Prematurity program (KIDROP), is a tele-medicine network that screens for ROP in different zones of Karnataka state in rural India. North Karnataka is the most underdeveloped and remote zone of this program and did not have any ROP screening programs before the intervention of the KIDROP in 2011. Six government and eleven private neonatal centers in this zone were screened weekly. Specific neonatal guidelines for ROP were developed and introduced in these centers. They included awareness about risk factors, oxygen regulation protocols, use of pulse oxymetry, monitoring postnatal weight gain, nutritional best practices, and management of sepsis. The incidence and severity of ROP were compared before the guidelines were introduced (Jan 2011 to Dec 2012) and after the guidelines were introduced (July 2013 to June 2015).

Results: During this 4-year period, 4,167 infants were screened over 11,390 imaging sessions. The number of enrolled infants increased from 1,825 to 2,342 between the two periods $(P<0.001)$. The overall incidence of any stage ROP reduced significantly from $26.8 \%$ to $22.4 \%$ $(P<0.001)$. The incidence of treatment-requiring ROP reduced from $20.7 \%$ to $16 \%(P=0.06)$, and of the treated disease, aggressive posterior ROP reduced from $20.8 \%$ to $13.1 \%(P=0.23)$ following introduction of the guidelines.

Discussion: Rural neonatal centers in middle-income countries have a large, unscreened burden of ROP. Improving neonatal care in these centers can positively impact the incidence and severity of ROP even in a relatively short period. A combined approach of a robust ROP screening program and improved neonatal care practices is required to address the challenge.

Keywords: KIDROP, preventive care, rural, incidence, laser photoablation, tele-medicine

\section{Introduction}

Middle-income countries (like India) are currently suffering from the "third epidemic" of retinopathy of prematurity (ROP). ${ }^{1,2}$ Over the past 2 decades, owing to improved neonatal care, infant mortality in India has considerably reduced, leading to an increased survival of these infants. Furthermore, the incidence of premature babies surviving has increased manifold. The World Health Organization reported in 2012 that 3.5 million infants are born premature in India annually, more than any other nation in the world. ${ }^{3}$

With $>60 \%$ of the nation's population still residing in rural areas combined with the improved survival of babies even in rural hospitals, the "at-risk" population for ROP in rural India has become a challenge to manage. Furthermore, the number of ROP 
specialists in India is dismally low. Although the absolute number and expertise of ROP specialists are currently unknown, it is believed that there are $<100$ such specialists, almost all of whom practice in urban centers. This leaves a large segment of the rural premature infants at risk of unscreened and untreated ROP blindness. ${ }^{4}$

To address this unmet challenge, the Karnataka Internet Diagnosis of Retinopathy of Prematurity (KIDROP) was initiated in 2008 with the purpose of employing trained and accredited nonphysicians to screen in remote and rural outreach centers. The salient features of this program include the use of wide-field digital imaging to screen at-risk infants in their neonatal care units, reporting on-site using a decisionbased algorithm, and relying on an indigenously developed tele-medicine network. ${ }^{5,6}$ There is a short turnaround cycle of receiving reports from ROP specialists situated in the city, who use their smart phone to report and make the decision accessible to these technicians. ${ }^{7}$

We have previously reported data from our multicenter rural program from two geographically defined zones, North and Central Karnataka. ${ }^{6}$ The report included data from 7,106 premature infants screened in 36 rural neonatal centers, over 77 months of activity. The incidence of treatment-requiring ROP was $3.57 \%$ of the babies screened. However, large regional variations between the zones were observed. In this paper, we describe the declining incidence and improving profile of the type of ROP in the north zone over a 4-year period following introduction of neonatal care guidelines that were introduced in this zone after the first 2 years and the impact of the same in the latter 2 years of the study.

\section{Methods}

\section{ROP screening}

The ROP screening program has been previously described in detail. 5,6 Briefly, the service of the KIDROP program in the north and central zones is under the aegis of a publicprivate partnership with the National Rural Health Mission (NRHM), Ministry of Health and Family Welfare, Government of Karnataka, since 2009. The region for this study, ie, the North Karnataka zone, has six districts, namely, Raichur (headquarters), Gulbarga, Bidar, Bijapur, Bagalkot, and Koppal. The Institutional Review Board, the Research Committee, and the Ethics Committee of the Narayana Nethralaya Postgraduate Institute of Ophthalmology, Bangalore, approved this program.

Active screening in this zone began in February 2011. Prior to this, in 2010, a specialized team comprising a technician and a project manager were trained at the headquarters in
Bangalore. Our training methods, scoring, and criteria for qualifying to be certified as a Level 3 technician have been previously published. ${ }^{5}$ A Level 3 technician captures images on the RetCam Shuttle and can decide on follow-up based on a three-way algorithm - "requires treatment", "requires follow-up", and "can be discharged". The decisions agreed with $94.3 \%$ of those of an ROP specialist, with the Level 3 technician missing only $0.4 \%$ of those needing treatment. ${ }^{5}$ The NRHM provides financial and logistic support of the recurring costs of the team, although the private hospital (Narayana Nethralaya Postgraduate Institute of Ophthalmology) managing the program offers screening and treatment at no cost. The team travels on a fixed schedule and visits one district each day of the week.

In the study zone, the team visits six public and eleven private hospitals weekly. Infants born $<2,000 \mathrm{~g}$ at birth and/or $<34$ weeks of gestation were enrolled into the study in accordance with the national ROP guidelines. ${ }^{8}$ At each session, technicians performed the "KIDROP sequence" of image acquisition for each infant. This included a minimum of seven images per eye. ${ }^{9}$ Standard ROP screening guidelines were followed to determine the follow-up. ${ }^{8}$ Treatment in the program was performed by laser photoablation using the Early Treatment for Retinopathy of Prematurity guidelines with the $532 \mathrm{~nm}$ green laser. ${ }^{10}$ As far as possible, the treatment was performed at the rural center by the ROP specialist traveling from Bangalore, thereby obviating the need for the infant to travel to the city. In those cases where this was not possible, the baby was transferred to Bangalore for treatment.

\section{Data review}

The diagnoses of the infants screened are shared with the treating neonatologists of the respective centers. A register is maintained in each neonatal intensive care unit (NICU) and is updated at each visit. In addition, the team maintains a data register in hard copy and online. All images are stored and archived on a secure server dedicated for this program. Furthermore, images of the babies are also shown to their respective mothers and other decision makers of the family at the end of the imaging session wherever possible. This is always done for babies who require treatment. This enhances family participation and improves compliance of follow-up. Data of any NICU are not shared with any other center, and patient information is anonymized in all cases.

Every month a summary of the data with a detailed review of the incidence of any stage ROP, treatment-requiring ROP (Type 1), and aggressive posterior ROP (APROP) is computed and submitted to the NRHM. Every quarter, 
there is a more detailed review of the data and suggestions to improve the program. Every 6 months, there is a review with the government where feedback on improving the enrollment and expansion of the program is discussed. Constant feedback on the incidence and severity of ROP in each center is discussed with the treating neonatologists or pediatricians.

The program director visits the NICUs periodically and discusses measures to reduce the incidence of ROP. Knowledge sharing and skill transfer activities, including sharing best care practices in neonatal care, training and retraining of the nursing staff and the paramedical staff encountered during the ROP screening, sharing publications, reports, and good clinical practice guidelines with the NICU administrators and doctors, increasing awareness among the mothers to act as ambassadors to educate other potential mothers in their villages about ROP, promoting good newborn care practices, including breast-feeding and kangaroo mother care, and promoting health education about immunization are carried out in several sessions.

\section{Improving neonatal care practices}

Centers that had the highest incidence of APROP were specially targeted to promote best neonatal care practices in an attempt to reduce severe ROP. These guidelines have been laid down by a special ROP subcommittee of the national neonatology foundation, Karnataka state chapter, and published elsewhere. ${ }^{11}$

A summary of these guidelines includes the following:

- Reducing iatrogenic premature births as far as possible. We promoted the discussion between the obstetrician and the neonatologist regarding the timing of the neonatal birth and promoted the use of antenatal steroid injection in the mother.

- Promoting the judicious use of oxygen - which included using pulse oxymetry soon after birth, during resuscitation, transport into the NICU or to another facility, and during the stay in the NICU.

- Avoiding the use of oxygen, especially $100 \%$ and unblended soon after birth especially if there were spontaneous respiratory efforts.

- Promoting the concept of fraction of inspired oxygen $\left(\mathrm{FiO}_{2}\right)$ to resuscitate preterm infants. This was not possible in all cases because of the lack of equipment in these rural centers.

- Removal of the reservoir from the bag to ventilate the baby when using bag and mask for positive-pressure ventilation ensuring the $\mathrm{FiO}_{2}$ of $<0.40$.
- Altering the $\mathrm{FiO}_{2}$ based on the pulse oxymetry readings.

- Promoting the concept of target saturation $\left(\mathrm{SaO}_{2}\right)$ between $88 \%$ and $92 \%$.

- Avoiding large fluctuations of $\mathrm{FiO}_{2}$ during oxygen desaturation episodes.

- Not to turn off the desaturation alarms in the NICU, especially at night.

- Promoting good postnatal weight gain and promoting the concept of total parenteral nutrition. Starting enteral feeds early as soon as the baby's condition is stable.

- Preventing late-onset sepsis with strict aseptic protocols and intravenous fluid handling.

- Pasting printed guidelines for ROP screening on the NICU wall and constant retraining of all shifts of nurses attending the NICU.

A majority of these guidelines were introduced in mid2013. We reviewed the incidence of the profile of ROP in the first 2 years of the program (2011-2013) and compared it with the profile 2 years (2013-2015) later to study the effect of these practices on the incidence and severity of ROP in these centers.

All statistical analysis was done using MedCalc Statistical Software Version 15.6.1 (MedCalc Software bvba; MedCalc, Ostend, Belgium; https:/www.medcalc.org; 2015). A proportion statistics was applied to analyze the data in the two study periods and to determine the difference between them.

\section{Results}

The study period was from January 2011 to June 2015. The details of the overall study cohort are summarized in Table 1, and their demographic details are summarized in Table 2. During this 4-year period, 4,167 infants were screened in 11,390 imaging sessions and 158,406 RetCam images were analyzed. The mean birth weight of babies enrolled in the two periods and the mean periods of gestation were 1,592.7 and 1,578.8 $\mathrm{g}$ and 31.7 and 31.6 weeks, respectively; both were comparable $(P>0.05)$.

Table I Demographics of the study zone

\begin{tabular}{ll}
\hline Parameter & Value \\
\hline Zone & North Karnataka \\
Number of districts & Six \\
Number of neonatal intensive care & I7 (six public and eleven private \\
units (NICUs) & NICUs) \\
Total babies screened & 4,167 babies (2, I72 public and \\
& I,995 private) \\
Study period & January 20II to July 20I5 \\
Imaging sessions & II,390 \\
Images & $I 58,406$ \\
\hline
\end{tabular}


Table 2 Birth weight and gestational age distribution of the study cohort

\begin{tabular}{ll}
\hline Parameter & Value \\
\hline Number of babies & 4,167 \\
Sex distribution (M:F) & $2,292: \mathrm{I}, 875$ \\
Birth weight distribution (N, \% of total) & \\
$\leq \mathrm{I}, 000 \mathrm{~g}$ & $\mathrm{I} 75(4.2)$ \\
$\mathrm{I}, 00 \mathrm{I}-\mathrm{I}, 500 \mathrm{~g}$ & $\mathrm{I}, 683(40.4)$ \\
$\mathrm{I}, 50 \mathrm{I}-\mathrm{I}, 750 \mathrm{~g}$ & $\mathrm{I}, 009(24.2)$ \\
$\mathrm{I}, 75 \mathrm{I}-2,000 \mathrm{~g}$ & $867(20.8)$ \\
$\geq 2,00 \mathrm{I} \mathrm{g}$ & $433(10.4)$ \\
Gestational age distribution (N, \% of total) & \\
$\leq 30$ weeks & $\mathrm{I}, \mathrm{I} 79(28.3)$ \\
$3 \mathrm{I}-32$ weeks & $979(23.5)$ \\
$33-34$ weeks & $\mathrm{I}, 042(25.0)$ \\
$\geq 35$ weeks & $967(23.2)$ \\
\hline
\end{tabular}

Abbreviations: $M$, male; $F$, female.

The incidence of any stage ROP during the 4-year period was $24.5 \%$ and of treatment-requiring ROP was $4.5 \%$. The overall distribution of the disease profile is summarized in Table 3 and the disease burden between the two study periods in Table 4. The number of enrolled neonatal centers remained constant throughout both periods; however, the number of enrolled infants increased from 1,825 to 2,342 between the two periods $(P<0.001)$. The overall incidence of any stage ROP reduced significantly from $26.8 \%$ in the first 2 years when compared to $22.4 \%$ in the latter 2 -year period $(P<0.001)$. The number of babies undergoing treatment also reduced between the two study periods from 101 (20.7\% of those with ROP) to 84 (16\% of those with ROP), but this was not statistically significant $(P=0.06)$.

Similarly, the number of babies with APROP reduced from 21 infants (20.8\% of those treated) to eleven infants $(13.1 \%$ of those treated) $(P=0.23)$. Although this difference was not

Table 3 ROP distribution in the study cohort

\begin{tabular}{ll}
\hline Parameter & Value, N (\%) \\
\hline No ROP & $3,146(75.5)$ \\
Any stage ROP & $1,02 I(24.5)$ \\
Treatment-requiring ROP & I85 (4.4) \\
Stage of ROP distribution (n=I,02I) & $\mathbf{N}(\%$ proportion of disease) \\
Stage I & $40 I(39.3)$ \\
Stage 2 & $435(42.6)$ \\
Stage 2 with Plus & $2 I(2 . I)$ \\
Stage 3 & $132(12.9)$ \\
Stage 4 & $0(0)$ \\
Stage 5 & $0(0)$ \\
APROP & $32(3.1)$ \\
\hline
\end{tabular}

Abbreviations: ROP, retinopathy of prematurity; APROP, aggressive posterior ROP.
Table 4 ROP distribution compared during the two study periods

\begin{tabular}{|c|c|c|c|}
\hline Parameter & $20 I I-2013$ & $2013-2015$ & Total/P-value \\
\hline $\begin{array}{l}\text { Number of } \\
\text { babies screened }\end{array}$ & I,825 & 2,342 & $4,167 / P<0.001$ \\
\hline $\begin{array}{l}\text { Number of } \\
\text { imaging sessions }\end{array}$ & 5,566 & 5,824 & 11,390 \\
\hline $\begin{array}{l}\text { Number of } \\
\text { babies with any } \\
\text { stage ROP }\end{array}$ & 489 (26.8\%) & 525 (22.4\%) & $P<0.001$ \\
\hline $\begin{array}{l}\text { Incidence of } \\
\text { type } 2 \text { ROP }\end{array}$ & 388 (79.3\%) & $44 \mathrm{I}(84 \%)$ & \\
\hline $\begin{array}{l}\text { Number of } \\
\text { babies requiring } \\
\text { treatment }\end{array}$ & IOI (20.7\%) & $84(16 \%)$ & $P=0.06$ \\
\hline $\begin{array}{l}\text { Number of } \\
\text { babies with } \\
\text { APROP }\end{array}$ & 21 (20.8\%) & II (I3.I\%) & $P=0.23$ \\
\hline
\end{tabular}

Abbreviations: ROP, retinopathy of prematurity; APROP, aggressive posterior ROP.

statistically significant, clinically, there was an improvement in the type and severity of the disease observed (Figure 1). In the first study period, all babies with APROP (21/21 babies) had posterior zone 1 disease with large capillary nonperfusion retinal beds extending irregularly like "tongues" into the central macula and accompanied by very severe plus disease (Figures 2 and 3). In the latter study period, 36.4\% (4/11) were in zone 1 , while the remaining $(7 / 11)$ had less severe presentation with the disease observed in zone 2 posterior (Figures 4 and 5). Following laser photoablation with $532 \mathrm{~nm}$ Nd:YAG laser (Iridex, CA, USA), five out of 42 eyes (11.9\%)

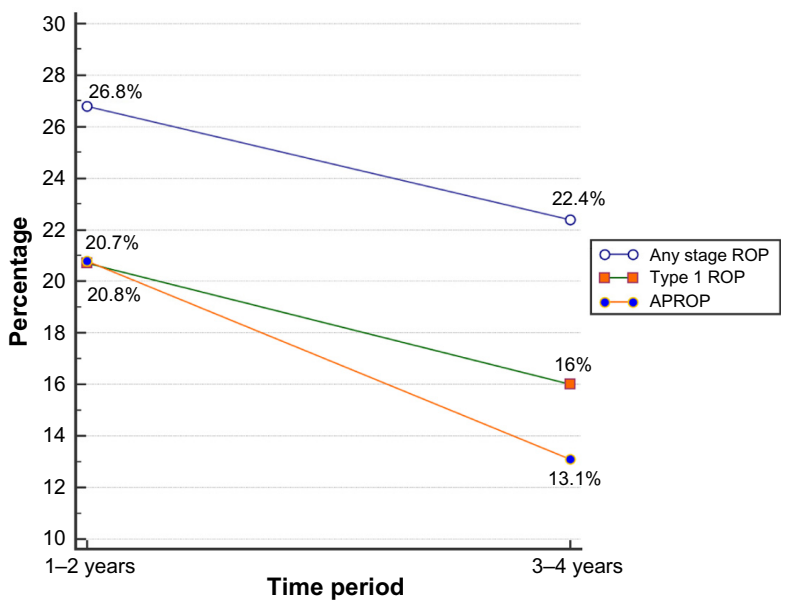

Figure I The graph depicts the trend of decline in the disease profile over the first 2 years and the latter 2-year period following the introduction of better neonatal care practices.

Notes: There was a decline in the incidence of any stage ROP, treatment-requiring ROP, and APROP but only the first category was statistically significant.

Abbreviations: ROP, retinopathy of prematurity; APROP, aggressive posterior ROP. 


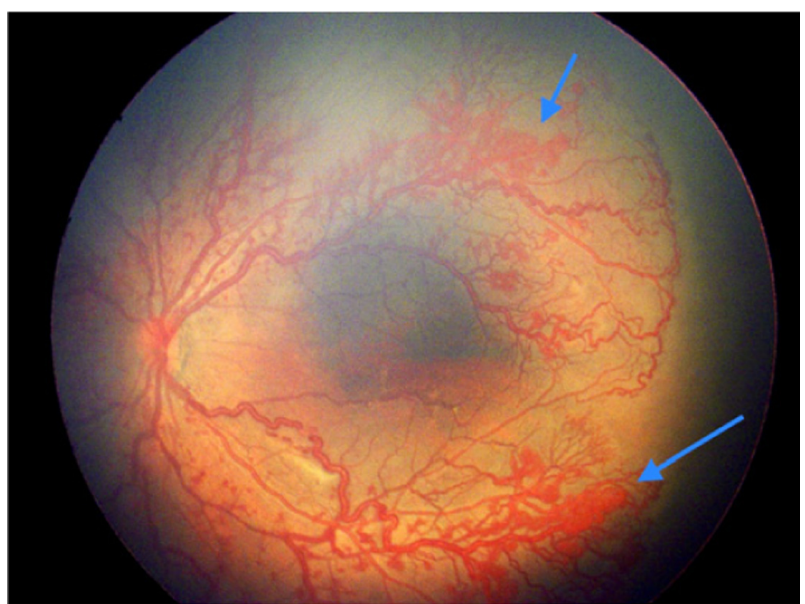

Figure 2 Left eye of a female infant born at 33 weeks weighing I,750 g in a private rural neonatal center showing aggressive posterior retinopathy of prematurity with neovascularization in zone I (blue arrows), severe plus disease, and closed capillary loops.

treated in the first period progressed to unfavorable outcome compared to $0 \%$ in the second period. Overall, $92.2 \%$ had a successful outcome.

\section{Discussion}

ROP is one of the leading causes of preventable infant blindness in the world. With improving neonatal care, upper ${ }^{12-21}$ and lower ${ }^{22-30}$ middle-income countries are reporting an incidence of severe ROP as high as $44 \%$. A summary of ROP incidence in some of these countries is detailed in Table 5.

ROP in rural India has recently been reported and appears to contribute to a significant burden of infant blindness.

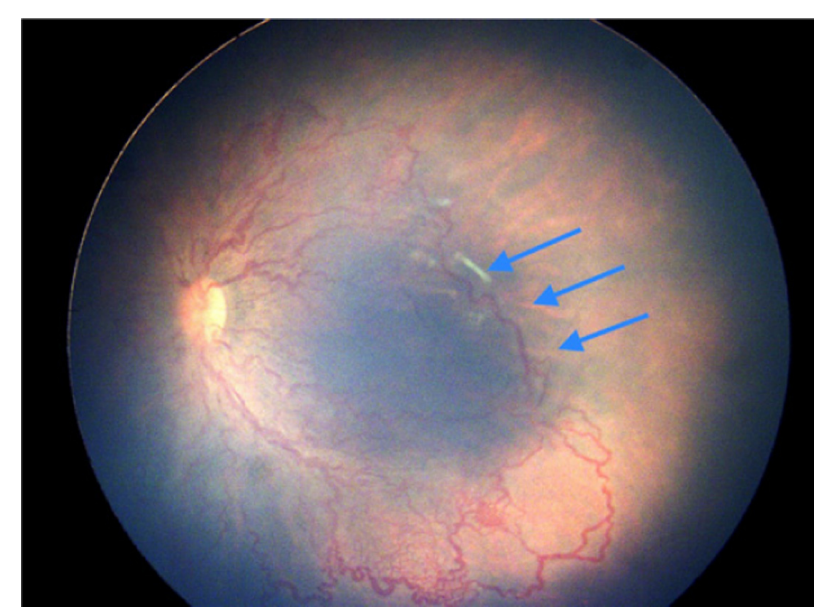

Figure 3 Left eye of a male infant born at 32 weeks weighing I,300 $\mathrm{g}$ in a rural center showing occluded and closed loops of vessels abruptly stopping in posterior zone I.

Note: The anterior extent of these loops ends before the temporal border of zone I (blue arrows).

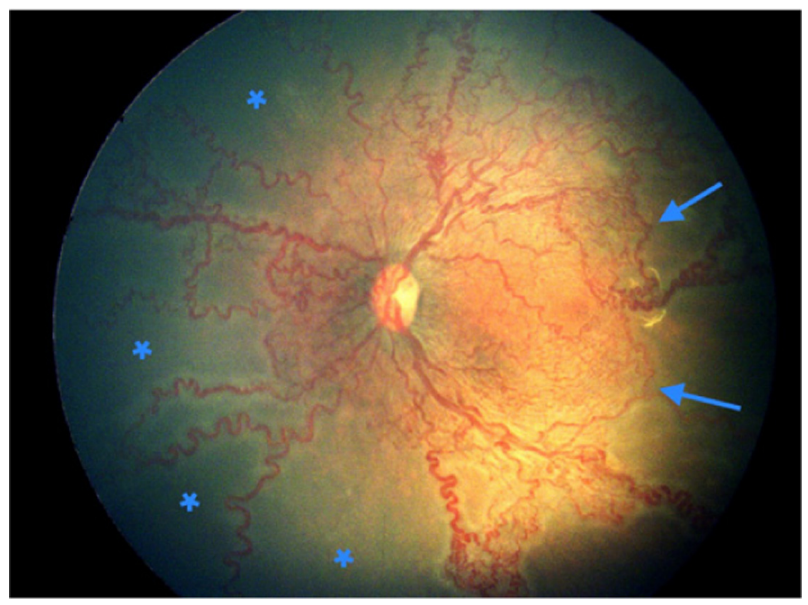

Figure 4 Left eye of a female infant born at 32 weeks weighing I,400 g shows aggressive posterior ROP.

Notes: There are capillaries only in the posterior pole (blue arrows). The anterior extensions of the dilated vasculature beyond contain ischemic, "bald" capillary devoid zones (blue asterisks) and extend into zone 2.

Abbreviation: ROP, retinopathy of prematurity.

Though it has been reported from urban centers for over 2 decades in India, ${ }^{22-24,31-34}$ rural data had been scarce. ${ }^{5,6,27,35}$ The first prospective study of rural ROP in 2012 reported an overall incidence of $41.5 \%$ and treatment-requiring incidence of $10.2 \% .{ }^{27}$ More recently, a multicenter rural study revealed an overall incidence of $22.39 \%$ and $3.57 \%$ requiring treatment. ${ }^{6}$ While APROP in heavier babies in urban settings is known, ${ }^{33,36}$ it has more recently been reported in rural centers with a high variation of up to $13 \%{ }^{37}$

The demographics of babies admitted in rural NICUs in our setting suggest that $4 \%$ of admissions were $<1,000 \mathrm{~g}$ at birth and $29 \%$ were $<30$ weeks of gestation, indicating that smaller and lighter babies are increasingly surviving in rural centers. ${ }^{6}$ This indicates an overall improvement in the general neonatal care. However, even within the same state, there are large variations in the ROP profile. Comparing two adjoining zones in Karnataka state, the incidence of any stage ROP was reported as $24.5 \%$ vs $19.6 \%$ and the treatmentrequiring disease was $4.5 \%$ vs $2.4 \%$, despite the ethnic and demographic features of the two zones being comparable. This allows speculation on the factors that may influence the severity of ROP in rural settings. Rural data have also shown that a significantly larger proportion of babies needing ROP treatment were from private hospitals rather than public hospitals ( $7.1 \%$ vs $1.7 \%){ }^{6}$ It is possible that this gap could be attributed to aggressive neonatal management in the private hospitals since there may be a compulsion to improve survival. However, this has not been confirmed in any formal study till date. 


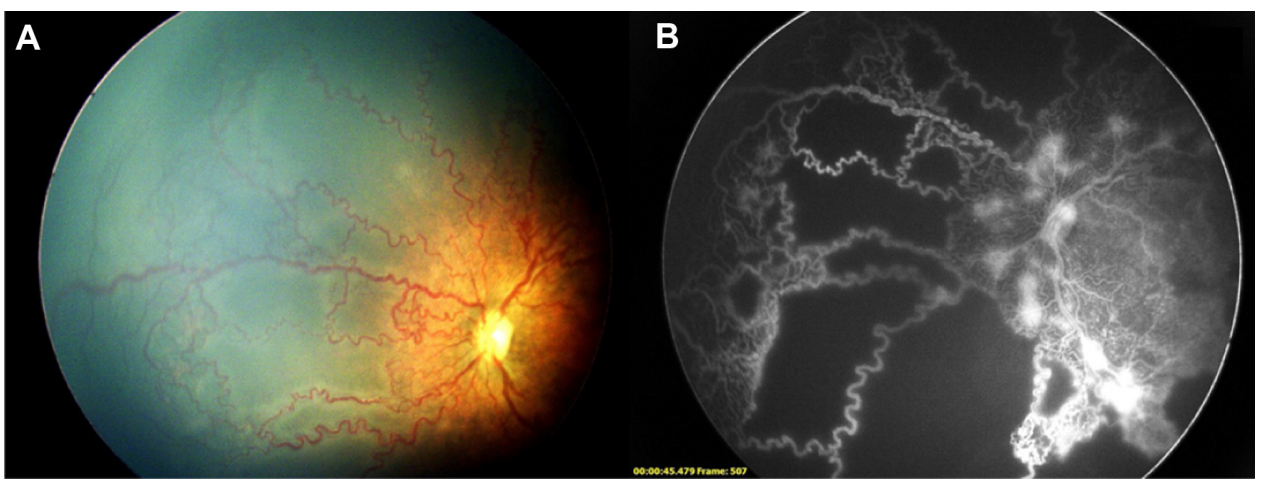

Figure 5 (A) Left eye nasal quadrant of a male baby born at 33 weeks weighing I,500 g shows ischemic loops enclosing areas of capillary drop out extending until the anterior edge of zone 2. (B) The angiogram on the same eye confirms the capillary non-perfusion within these loops. Leaking neovascularization adjacent to the optic disc is also noted.

The most striking difference encountered between rural zones reported thus far is the skewed distribution of APROP between two adjoining areas of the same state. Over $84 \%$ of all reported cases of APROP from a state came from one zone compared to only $16 \%$ from another adjoining zone. ${ }^{6}$ All other factors being comparable between the two zones, the cause of this large difference could be attributed to the level of neonatal care. The zone with the higher incidence of APROP (North Karnataka) is the subject of this current manuscript.

APROP in India affects "heavier" and "larger" babies compared to their western counterparts. ${ }^{32,33,36,37}$ Shah et al reported the similarity of the APROP seen in India with the oxygen-induced retinopathy models in animals. This was due to the injudicious use of unblended, high-volume oxygen in these babies. ${ }^{32}$ Interestingly, the 21 cases of APROP seen in the first 2 years of this study resembled the cases described by Shah et al in location and severity. Indeed, site visits to these "APROP centers" revealed poor understanding and practice of oxygen delivery and general neonatal risk factor management. In one such center, the nurse had been discharged from duty and an unqualified paramedic who would switch off the pulse oxymetry alarms at night and administer $100 \%$ oxygen to babies admitted in the NICU took her place. It became imperative for us to address neonatal care practices besides providing regular ROP screening services at these centers.

Table 5 Incidence of ROP in upper and lower middle-income countries

\begin{tabular}{|c|c|c|c|c|c|}
\hline Country & Study & Period & $\begin{array}{l}\text { Number of } \\
\text { NICUs }\end{array}$ & $\begin{array}{l}\text { Number of } \\
\text { babies }\end{array}$ & $\begin{array}{l}\text { Severe ROP } \\
\text { incidence (\%) }\end{array}$ \\
\hline \multicolumn{6}{|l|}{ Upper middle-income countries } \\
\hline Argentina & Tavosnanska $^{12}$ & $2008-2010$ & I & 1,169 & 13 \\
\hline Brazil & Zin et $\mathrm{al}^{13}$ & 2004-2006 & 7 & 3,437 & 3.4 \\
\hline People's Republic of China & Li et $\mathrm{al}^{14}$ & $2009-2010$ & I & 2,185 & 1.3 \\
\hline Colombia & Zuluaga et al ${ }^{15}$ & $200 I-2005$ & 1 & 1,138 & 8.0 \\
\hline Iran & Saeidi et al ${ }^{16}$ & $2005-2006$ & I & 45 & 8.5 \\
\hline Lithuania & Jakuskiene et $\mathrm{al}^{17}$ & $2003-2005$ & I & 338 & 4.2 \\
\hline Romania & Vătavu et $\mathrm{al}^{18}$ & $2002-2007$ & I & $\mathrm{I}, 783$ & 15.2 \\
\hline Serbia & Knezevic et al ${ }^{19}$ & 2006-2009 & I & 478 & 21.3 \\
\hline South Africa & Delport et $\mathrm{a}^{20}$ & 1999 & 1 & 94 & 4.3 \\
\hline Turkey & Akkoyun et $a^{21}$ & $2002-2004$ & I & 88 & 29.5 \\
\hline \multicolumn{6}{|l|}{ Lower middle-income countries } \\
\hline India & Charan et $\mathrm{al}^{22}$ & 1993 & I & 165 & 12.8 \\
\hline India & Gopal et $\mathrm{a}^{23}$ & $1992-1994$ & I & 50 & 16.0 \\
\hline India & Vinekar et $\mathrm{al}^{24}$ & $1993-2003$ & I & 138 & 44.9 \\
\hline India & Varughese et $\mathrm{a}^{25}$ & $1999-2000$ & I & 79 & 6.3 \\
\hline India & Jalali et $\mathrm{a}^{26}$ & 1999-2002 & 2 & $\mathrm{I}, 083$ & 11.0 \\
\hline India & Hungi et $\mathrm{al}^{27}$ & $2008-2010$ & I & 118 & 10.2 \\
\hline Pakistan & Taqui et $\mathrm{a}^{28}$ & $2003-2006$ & I & 68 & 20.6 \\
\hline Vietnam & Phan et al ${ }^{29}$ & 2001 & I & 225 & 9.3 \\
\hline Bangladesh (low income) & Ahmed et $\mathrm{al}^{30}$ & 1998-2003 & I & 114 & 4.4 \\
\hline
\end{tabular}

Abbreviations: ROP, retinopathy of prematurity; NICUs, neonatal intensive care units. 
The guidelines for improved neonatal care that are described in this study were evolved with the purpose of providing simple recommendations that were aimed at addressing this lacunae in neonatal care practice. ${ }^{11}$ They were developed by the ROP subcommittee of the state and also addressed the pediatrician, given the reality that pediatricians and not formally trained neonatologists, owing to the scarcity of the latter in the country, manage most rural centers. By comparing the incidence and profile of ROP in these centers before and after the introduction of these guidelines, we aimed to study the impact of improved neonatal care practices in a rural zone, which had very severe ROP.

The study results bring out some interesting aspects. First, the number of babies enrolled for ROP screening increased between the two periods from 1,825 to 2,342 babies, respectively $(P<0.001)$. This occurred despite the number of centers and districts remaining constant. This could be attributed to more diligent referral from the study center pediatricians and by local pediatricians from smaller hospitals, which were not visited by our team. It must be emphasized that ROP screening was nonexistent in these centers before our program in 2011, and enhancing awareness resulted in an enhanced participation. Second, the incidence of any stage ROP decreased between the two periods ( $26.8 \%$ vs $22.4 \%$, $P<0.001)$. This occurred despite the fact that more babies were enrolled between the two periods. This positive trend could be attributed to an overall improvement in neonatal care, stricter adherence to the ROP screening guidelines, ${ }^{8}$ and better control of risk factors. Third, the incidence of treatment-requiring ROP (101 vs 84 babies) and APROP (21 vs 11 babies) showed a decline in the later 2 years. Although this was not significant statistically, this decline is of clinical importance. Fourth, the type of severe disease showed a change in the morphology from a more severe form to a less severe one. All cases of APROP in the first period were in zone 1 and resembled the oxygen-induced retinopathy model described by Shah et al, ${ }^{32}$ with occluded vessels, posterior extension of the ischemic tongues, and severe plus disease (Figures 2 and 3). In the second period, four of eleven babies $(36 \%)$ were in zone 1 and the remaining cases of APROP showed a less severe form (Figures 4 and 5). This change in morphology highlights the importance of better oxygen delivery management, which was an important aspect of the guidelines. Finally, these changes, that is, the decline of incidence and severity of ROP occurred in a relatively short period of 4 years.

This report differs from others in the study duration, number of infants screened, and the disease trends. While most previous reports have shown an increase in the disease burden over an 8- to 10 -year period, ${ }^{38-41}$ one study has found a significant decrease in ROP during the second period. ${ }^{42}$ We found a decline in the disease incidence over a shorter time period of 2 years, despite a larger number of infants screened. This could be attributed to the impact this program has had in an area where no ROP screening previously existed. The critical level of change in risk factor modifications required to create a significant difference in outcome would appear to be less, contrasted to an area where ROP screening was already in practice. In the next few years, it is likely that a further reduction in the disease profile will take longer and with more extensive intervention-based modifications.

The limitation of the study lies in the fact that we do not have a quantitative estimate on which neonatal practice contributed in what measure. We did not undertake objective measurements of the level of knowledge or skill before and after the guidelines were introduced. In addition, we did not assess the knowledge, attitude, and practice among the pediatricians or neonatologists who were introduced to these practices. However, subsequent site visits have documented an improvement in equipment and protocols being followed in these centers. Indeed, not all centers are equally amenable to change, and we have attempted to classify "supportive" and "nonsupportive" neonatal centers in these rural areas. ${ }^{43}$ A formal test of knowledge and assessment of the extent of practices adopted needs to be evaluated. Finally, given the occurrence of high variability in the infant mortality rates and neonatal care practices in different states and zones of the country, our results in rural Karnataka cannot be extrapolated to other states without considering regional demographics and further operational research.

\section{Conclusion}

The experience from our rural work has become the subject of the national task force for ROP constituted by the federal government (National Health Mission, Government of India). In the ROP management guidelines that are being prepared, neonatal care practices that promote the prevention of severe ROP are being actively considered. This includes training of nursing and paramedical staff of the district-based, special newborn care units. The role of the treating pediatrician or neonatologist has also been highlighted. Observing the cohort prospectively will address this concern.

With the expansion of neonatal care services throughout the country, the special newborn care units envisaged by the federal government to be setup in each district of the country are going to add a very large load of at-risk babies for ROP screening. ${ }^{44}$ The role of the pediatrician, neonatologist, and the obstetrician has become paramount. The current 
infrastructure is equipped neither with trained manpower nor with essential technology to tackle this challenge. Integrating training of the neonatal staff and physicians with best care practices would help reduce the incidence of severe ROP. The National Task Force for ROP in India in collaboration with the National Neonatology Foundation of India is preparing training modules for pediatricians and nurses to address these lacunae.

\section{Acknowledgments}

We acknowledge the contributions of the National Neonatology Foundation, Karnataka state chapter, subcommittee for ROP members, Dr Archana Bilagi, Dr Ranjan Pejaver, Dr Sangappa Dhaded, and Dr Ravindra Battu for their help in framing the guidelines and the KIDROP team members of North Karnataka, Mr Muralidhar Gayakwad, Mr Ravishankar Kandagal and Bangalore team members Mr Praveen Sharma, Mr Sivakumar Munusamy, and Mr Krishnan for their support.

\section{Disclosure}

The authors report no conflicts of interest in this work.

\section{References}

1. Gilbert C, Rahi J, Eckstein M, O'Sullivan J, Foster A. Retinopathy of prematurity in middle-income countries. Lancet. 1997;350(9070): $12-14$.

2. Gilbert C, Fielder A, Gordillo L, et al; International NO-ROP Group. Characteristics of infants with severe retinopathy of prematurity in countries with low, moderate, and high levels of development: implications for screening programs. Pediatrics. 2005;115(5):e518-e525.

3. The Global Action Report on Preterm Birth [webpage on the Internet]. United Nations 2012. Born Too Soon. 13(5):393-396. Available from: http://www.who.int/pmnch/media/news/2012/201204_borntoosoonreport.pdf. Accessed June 26, 2015.

4. Vinekar A. IT - enabled innovation to prevent infant blindness in rural India: the KIDROP experience. J Indian Bus Res. 2011;3(2):98-102.

5. Vinekar A, Gilbert C, Dogra M, et al. The KIDROP model of combining strategies for providing retinopathy of prematurity screening in underserved areas in India using wide-field imaging, tele-medicine, non-physician graders and smart phone reporting. Indian J Ophthalmol. 2014;62(1):41-49.

6. Vinekar A, Jayadev C, Mangalesh S, Shetty B, Vidyasagar D. Role of tele-medicine in retinopathy of prematurity screening in rural outreach centers in India - a report of 20,214 imaging sessions in the KIDROP program. Semin Fetal Neonatal Med. 2015;20(5):335-345.

7. Vinekar A, Jayadev C, Bauer N. Need for telemedicine in retinopathy of prematurity in middle-income countries: e-ROP vs KIDROP. JAMA Ophthalmol. 2015;133(3):360-361.

8. Pejaver RK, Vinekar A, Bilagi A [webpage on the Internet]. National Neonatology Foundation's Evidence Based Clinical Practice Guidelines 2010. Retinopathy of Prematurity (NNF India, Guidelines); 2010: 253-262. Available from: http://aimaonline.org/iap-neochap-2013/uploads/ acd-corner/nnf_guidelines-2011.pdf. Accessed January 23, 2016.

9. Vinekar A, Jayadev C, Mangalesh S, et al. Comparing the outcome of single versus multiple session laser photoablation of flat neovascularization in zone 1 aggressive posterior retinopathy of prematurity: a prospective randomized study. Retina. 2015;35(10):2130-2136.
10. Early Treatment For Retinopathy Of Prematurity Cooperative Group. Revised indications for the treatment of retinopathy of prematurity: results of the early treatment for retinopathy of prematurity randomized trial. Arch Ophthalmol. 2003;121(12):1684-1694.

11. Vinekar A, Jayadev C, Bilagi A, Pejaver R, Dhaded SM, Battu R. Screening and prevention of ROP: practical pearls for the ophthalmologist and the pediatrician. Perinatology. 2014;15:97-99.

12. Tavosnanska J. Morbimortalidad de recién nacidos con menos de 1500 gramos asistidos en hospitales públicos de la ciudad de Buenos Aires. [Mortality and morbidity of very low birth weight newborn infants assisted in Buenos Aires public hospitals]. Arch Argent Pediatr. 2012;110(5):394-403. Spanish.

13. Zin AA, Moreira MEL, Bunce C, Darlow BA, Gilbert CE. Retinopathy of prematurity in 7 neonatal units in Rio de Janeiro: screening criteria and workload implications. Pediatrics. 2010;126(2):e410-e417.

14. Li Q, Wang Z, Li Y, et al. [Retinopathy of prematurity screening in 2185 premature infants]. Zhonghua Yan Ke Za Zhi. 2012;48(10):903-907.

15. Zuluaga C, Llanos G, Torres J. Effects of the screening program on ROP in Cali, Columbia. Acta Med Litu. 2006;13(3):176-178.

16. Saeidi R, Ahmad H, Ahmadi S, Rahmani S. Prevalence and predisposing factors of ROP in very low-birth-weight infants discharged from NICU. Iran J Pediatr. 2009;19(1):59-63.

17. Jakuskiene R, Vollmer B, Saferis V, Daugeliene D. Neonatal outcomes of very preterm infants admitted to a tertiary center in Lithuania between the years 2003 and 2005. Eur J Pediatr. 2011;170(10):1293-1303.

18. Vătavu I, Nascutzy C, Ciomârtan T, Brezan F, Anca I, Stoicescu S. [Retinopathy of prematurity--screening results]. Oftalmologia. 2010;54(1):110-117.

19. Knezevic S, Stojanovic N, Oros A, Savic D, Simovic A, Knezević J. Analysis of risk factors in the development of retinopathy of prematurity. Srp Arh Celok Lek. 2011;139(7-8):433-438.

20. Delport SD, Swanepoel JC, Odendaal PJL, Roux P. Incidence of retinopathy of prematurity in very-low-birth-weight infants born at Kalafong Hospital, Pretoria. S Afr Med J. 2002;92(12):986-990.

21. Akkoyun I, Oto S, Yilmaz G, et al. Risk factors in the development of mild and severe retinopathy of prematurity. J AAPOS. 2006;10(5): 449-453.

22. Charan R, Dogra MR, Gupta A, Narang A. The incidence of retinopathy of prematurity in a neonatal care unit. Indian J Ophthalmol. 1995;43(3):123-126.

23. Gopal L, Sharma T, Ramachandran S, Shanmugasundaram R, Asha V. Retinopathy of prematurity: a study. Indian J Ophthalmol. 1995;43(2):59-61.

24. Vinekar A, Dogra MR, Sangtam T, Narang A, Gupta A. Retinopathy of prematurity in Asian Indian babies weighing greater than 1250 grams at birth: ten year data from a tertiary care center in a developing country. Indian J Ophthalmol. 2007;55(5):331-336.

25. Varughese S, Jain S, Gupta N, Singh S, Tyagi V, Puliyel JM. Magnitude of the problem of retinopathy of prematurity. Experience in a large maternity unit with a medium size level-3 nursery. Indian J Ophthalmol. 2001;49(3):187-188.

26. Jalali S, Matalia J, Hussain A, Anand R. Modification of screening criteria for retinopathy of prematurity in India and other middle-income countries. Am J Ophthalmol. 2006;141(5):966-968.

27. Hungi B, Vinekar A, Datti N, et al. Retinopathy of prematurity in a rural neonatal intensive care unit in south India-a prospective study. Indian J Pediatr. 2012;79(7):911-915.

28. Taqui AM, Syed R, Chaudhry TA, Ahmad K, Salat MS. Retinopathy of prematurity: frequency and risk factors in a tertiary care hospital in Karachi, Pakistan. J Pak Med Assoc. 2008;58(4):186-190.

29. Phan MH, Nguyen PN, Reynolds JD. Incidence and severity of retinopathy of prematurity in Vietnam, a developing middle-income country. J Pediatr Ophthalmol Strabismus. 2003;40(4):208-212.

30. Ahmed AS, Muslima H, Anwar KS, et al. Retinopathy of prematurity in Bangladeshi neonates. J Trop Pediatr. 2008;54(5):333-339.

31. Maheshwari R, Kumar H, Paul VK, Singh M, Deorari AK, Tiwari HK. Incidence and risk factors of retinopathy of prematurity in a tertiary care newborn unit in New Delhi. Natl Med J India. 1996;9(5):211-214. 
32. Shah PK, Narendran V, Kalpana N. Aggressive posterior retinopathy of prematurity in large preterm babies in south India. Arch Dis Child Fetal Neonatal Ed. 2012;97(5):F371-F375.

33. Jalali S, Kesarwani S, Hussain A. Outcomes of a protocol-based management for zone 1 retinopathy of prematurity: the Indian Twin Cities ROP Screening Program report number 2. Am J Ophthalmol. 2011;151(4):719-724.

34. Azad R. Prevention of blindness due to retinopathy of prematurity: a national movement. Indian J Pediatr. 2014;81(12):1373-1375.

35. Keerthi BJ, Babu S, Vinekar A, Goud N, Bullappa A. Retinopathy of prematurity screening of 500 infants in a level II neonatal intensive care unit at a medical college hospital in southern Karnataka. J Evol Med Dent Sci. 2014;3:10665-10672.

36. Sanghi G, Dogra MR, Das P, Vinekar A, Gupta A, Dutta S. Aggressive posterior retinopathy of prematurity in Asian Indian babies: spectrum of disease and outcome after laser treatment. Retina. 2009;29(9):1335-1339.

37. Vinekar A, Avadhani K, Braganza S, Shetty B, Dogra M, Gilbert C. Outcomes of a protocol-based management for zone 1 retinopathy of prematurity: the Indian twin cities ROP screening program report number 2. Am J Ophthalmol. 2011;152(4):712. author reply 713.

38. Schalij-Delfos NE, Cats BP. Retinopathy of prematurity: the continuing threat to vision in preterm infants. Dutch survey from 1986 to 1994 Acta Ophthalmol Scand. 1997;75(1):72-75.
39. Hameed B, Shyamanur K, Kotecha S, et al. Trends in the incidence of severe retinopathy of prematurity in a geographically defined population over a 10-year period. Pediatrics. 2004;113(6):1653-1657.

40. Schiariti V, Matsuba C, Houbé JS, Synnes AR. Severe retinopathy of prematurity and visual outcomes in British Columbia: a 10-year analysis. J Perinatol. 2008;28(8):566-572.

41. Demir S, Yücel ÖE, Niyaz L, Karakuş G, Arıtürk N. Incidence of retinopathy of prematurity in the middle Black Sea region of Turkey over a 10-year period. JAAPOS. 2015;19(1):12-15.

42. Gaugler C, Beladdale J, Astruc D, et al. [Retinopathy of prematurity: 10-year retrospective study at the University Hospital of Strasbourg]. Arch Pediatr. 2002;9(4):350-357.

43. Hosamani S, Kumar SVA, Vallabha K, Biradar SWV. Comparison of tele screening for retinopathy of prematurity screening and impact of neonatal team support in government and private centers in rural Bijapur district of Southern India. J Vis Sci. 2015;1(2):15-20.

44. Ministry of Health and Family Welfare [webpage on the Internet]. Operational Guidelines for Rashtriya Bal Swasthya Karyakram. Child Health Screening and Early Intervention Services Under NRHM. 2013. Available from: http://www.pbnrhm.org/docs/rbsk_guidelines.pdf. Accessed July 12, 2015.
Eye and Brain

\section{Publish your work in this journal}

Eye and Brain is an international, peer-reviewed, open access journal focusing on clinical and experimental research in the field of neuroophthalmology. All aspects of patient care are addressed within the journal as well as basic research. Papers covering original research, basic science, clinical and epidemiological studies, reviews and evaluations,

\section{Dovepress}

guidelines, expert opinion and commentary, case reports and extended reports are welcome. The manuscript management system is completely online and includes a very quick and fair peer-review system, which is all easy to use. Visit http://www.dovepress.com/testimonials.php to read real quotes from published authors. 